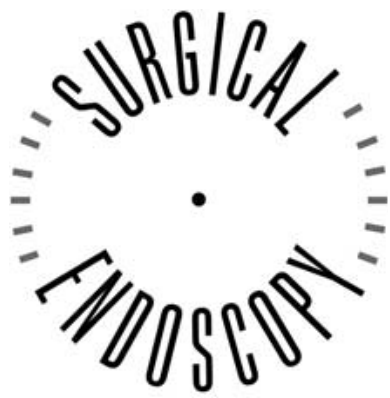

and Other Interventional Techniques

\title{
A simple device for measuring the resolution of videoscopic cam- eras and laparoscopes in the operating room
}

I read with great interest the report of Dr. Siperstein [1], in which he recommends the use of this relatively simple testing method by surgeons, operating room staff, and other clinicians to obtain some idea about the performance of a laparotelescope with an attached camera. It is a commendable idea. Currently, a plethora of various equipment is offered for the same application.

One major component of laparosurgery is the imaging system because we work from the television screen. The quality of the image, and whatever this means, is of great importance. To create a realistic available yardstick, we must be able to standardize the performance. This is almost as difficult as assessing the competence of the surgeon, which is now a "hot" issue. If we are discussing image quality of the optical and television system, we have to know about resolution, contrast, color reproduction, picture quality in the middle and at the edges of the image, depth of field, light sensitivity or light transmission, and viewing angle, to mention a few. In using this bench model, it must be possible to calibrate or standardize the light source for identical performance in each test in terms of brightness level, homogenicity, and the like.

Currently, there are so many marketing gimmicks. In reading some of the brochures, I agree with Dr. Siperstein that some guidelines are required to make sure that you get what you require or what was promised. As early as 1969 [2], I tried to introduce performance tests, which were foreign to consumers, and became almost a laughingstock of the surgical community.

Another logistical question-who should perform these tests. The surgeon is busy in the operating room and has many other commitments. The operating room personnel work on a very tight schedule. The purchasing officer is concerned about the lower bids in most cases and not in the quality performance. Another problem to be solved applies to telescope other than the $0^{\circ}$ direction of view.

With regard to testing, is resolution as one factor, enough? Light sensitivity of a camera is crucial as well as the absorption factor of the optics. What about color reproduction? Again, the idea of having some testing that is more objective than the Individual operator's observation on the TV screen would be valuable. The screens must be adjusted optimally and remain the same for all the tests. The the same person trained for this test must do the evaluation.

Currently, the concept of "standardization" is a very sensitive issue, but we must have a measuring scale for comparing the various brands available. The author should be commended for starting the procedure, which probably is just as difficult as addressing other issues, such as assessing the competence of a surgeon. It will be an uphill battle, but it is worthwhile to start.

\section{References}

1. Berber E, Pearl JM, Siperstein AE (2002) A simple device for measuring the resolution of videoscopic cameras and laparoscopes in the operating room. Surg Endoc 16: 1111-1113

2. Berci G, Kont LA (1969) A new optical endoscopy with special reference to cytoscopy. Br J Urol 41: 564-571

\section{G. Berci}

Department of Surgery

Cedars-Sinai Medical Center

8700 Beverly Boulevard

Suite 8215, Los Angeles

CA 90048-1865, USA

Online publication: 7 May 2002 